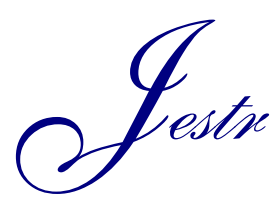

Research Article

\title{
CFD Simulation Analysis of Building Density on Residential Wind Environment
}

\author{
Kaihua $\mathrm{Hu}^{1,2}$, Shidan Cheng ${ }^{1, *}$ and Yi Qian ${ }^{1}$ \\ ${ }^{1}$ School of Urban Design, Wuhan University, Wuhan 430072, China \\ ${ }^{2}$ School of Architecture, Hubei Engineering University, Xiaogan 432000, China
}

Received 6 November 2017; Accepted 21 December 2017

\begin{abstract}
With the rapid urbanization and expansion of construction demands in China, the continuously increasing density of residential buildings has led to outdoor pedestrian discomfort in residential wind environment. To analyze the relationship between residential building density and wind environment, simulation cases of residential buildings with different building densities were presented in this study. First, the wind environment characteristics of residential districts with different building densities were established via computer numerical simulation. Second, wind speed ratio (WSR) and age of air (AA) distribution were calculated at pedestrian height. Finally, the quantitative relationship between residential outdoor wind environment and building density was proposed. Results demonstrate a negative linear correlation between the building density and outdoor mean wind speed ratio (MWSR) of residential districts and a positive linear relationship between building density and mean age of air (MAA). When residential building density increased from 0.18 to 0.32 , the MWSR in the district decreased by 0.18 , whereas MAA increased by $58.63 \mathrm{~s}$. As residential building density increases, the WSR within the residential district decreases and the age of air (AA) increases, which affect the outdoor comfort of residents. This study provides references for improving the wind environment of high-density residential districts and for optimizing residential layout planning.
\end{abstract}

Keywords: Wind environment; Residential buildings; Building density; CFD

\section{Introduction}

With the rapid urbanization and expansion of construction demands in China, the land use forms of high building density and building floor area ratio have become the solutions for the conflict between intense urban land use and dense urban population. Although high-rise buildings can be beneficial for the economical use of land to a certain extent, an excessively high building density makes the urban environment increasingly polluted and the ecological climate increasingly harsh. The high construction rate in a city has changed the original urban microclimate environment and may adversely affect the ventilation of surrounding buildings and the physical environment of other buildings [1, 2]. Highdensity residential districts will lead to the deterioration of residential wind environment, thereby resulting in wind currents, wind shadow areas, poor ventilation, and other adverse weather phenomena, which considerably affect the thermal comfort of people and even cause pedestrian safety problems [3].

In areas with hot summers and cold winters, such as Wuhan City in China, natural ventilation can eliminate excessive heat in summer and reduce the negative impact of humidity on people, thereby improving their thermal comfort [4]. In addition, a good residential wind environment can supply residents with fresh air, which is beneficial for their physical and mental health. A good wind

\footnotetext{
*E-mail address: chsd821@163.com

ISSN: 1791-2377 @ 2018. Eastern Macedonia and Thrace Institute of Technology. All rights reserved.

doi:10.25103/jestr.111.05
}

environment is one of the important factors in building a comfortable and healthy green ecological settlement.

Researchers have conducted numerous studies on the impact of high-density settlements on outdoor wind environment [5-7], but the quantitative assessment of wind environment and the relationship between building density and wind environment have not yet been fully investigated. For example, in-depth research on building density and other factors that affect wind environment remains lacking. Therefore, the accurate prediction of the impact of residential building density on wind environment and the clarification of the quantitative relationship between building density and wind environment are urgent issues that should be addressed.

On the basis of the preceding analysis, the present study establishes hypothetical building models of residential districts through computer numerical simulation and analyzes the mean wind speed ratio (MWSR), mean age of air (MAA), and wind environment characteristics of residential districts to accurately investigate the quantitative relationship between residential building density and wind environment, and consequently, optimize wind environment in high-density residential districts and provide references for residential planning.

\section{State of the art}

At present, numerous studies have been conducted on residential wind environment, and strategies and planning guidelines for optimizing wind environment have been proposed. However, these studies have mainly focused on 
urban structure, architectural layout, and architectural details. Zhang et al. [8] used computational fluid dynamics (CFD) simulations and wind tunnel experiments to study wind environment around different building layouts. A research on three schemes for parallel building arrangement found that wind environment around buildings exhibits a significant relation to the layout and direction of buildings. A staggered layout helps introduce wind into the interior of a building, which improves natural ventilation. However, macro-level research on residential building density remains lacking. Ying et al. [9] studied wind environment groups with six different layouts via numerical simulation. Their results showed that the O-type layout has the best outdoor wind environment. However, they did not study building density, which influences wind environment. Gao et al. [10] used statistical analysis methods to study the influence of urban architecture on wind field morphology. They concluded that meteorological data and building layout factors are the key factors that affect wind environment. Gong [11] used four typical layouts of urban settlements, namely, determinant, staggered, skewed, and mixed, as research objects, He found that the length of a building in residential wind environment is significant and the use of an oblique column layout can effectively improve wind environment. Fang et al. [12] used wind tunnel tests and numerical simulations to study the wind environment of highly simplified buildings in Lujiazui area, Shanghai. They pointed out that along the flow direction, high WSR zones exist on both sides of windward buildings and between two buildings, whereas low WSR zones exist upstream and downstream of buildings. Feng Yang [13] investigated the subjective perceptions of the wind comfort and thermal comfort of pedestrians in different types of settlements in Shanghai via field surveys and questionnaires. This study indicates that site planning and residential design should be dominated by summer ventilation, and winter wind protection may not be as relevant as summer ventilation. Elshafei et al. [14] introduced experimental and numerical studies to assess the effect of natural ventilation on thermal comfort inside residential buildings. A research proposed that window design parameters, such as window size and position, should be modified to improve indoor thermal comfort. Xing Shi et al. [15] demonstrated the application of the wind environment assessment method by using an actual case of urban planning as an example. The evaluation criteria included wind comfort, wind safety, and wind amplification factor. Their study provides a reference for assessing pedestrian wind environment in the urban planning and design phases. $\mathrm{Li}$ Tang [16] studied the wind environment of historical settlements in China by using the CFD method. This researcher evaluated the construction experience of a village in site selection, layout, and landscape adaptation to wind environment, but did not study the impact of building density on village wind environment. Tsang [17] studied high-rise buildings that affect pedestrian wind environment and reported that high and wide buildings have an adverse impact on wind environment. Macroeconomic research has not been conducted. Mochida [18] studied the significant impact of tree and vehicle canopy models on turbulent flow in a street canyon. Jones [19] introduced wind tunnel scale modeling and the numerical calculation of pedestrian wind environment. The aforementioned references indicate that CFD in actual wind environment requires further verification. Moreover, factors that affect wind environment have not yet been studied.

The previous research results have mainly focused on the study of the morphological layout of residential wind environment and the ventilation detail of residential buildings. However, only a few studies have addressed macroscopic factors, such as building density, particularly the quantification research of residential building density and wind environment. In the present study, simulation models of building density and wind environment in residential districts are established using computer numerical simulation and data statistics. The mean wind speed ratio (MWSR) and mean age of air (MAA) are used as evaluation indexes to discuss the wind environment of residential districts under different building density cases. The quantitative relationship between residential building density and wind environment is investigated, which provides references for residential planning and the improvement of residential wind environment.

The rest of this study is organized as follows. Section 3 describes the hypothetical computational cases and constructs the calculation models with different building densities. In Section 4, wind environment analysis of the cases is performed via computer numerical simulations. The wind speed ratio (WSR) distribution, statistical distribution of age of air (AA), and strategy for improving wind environment in residential districts are obtained under different cases. Section 5 concludes the study and provides the relevant findings.

\section{Methodology}

\subsection{Description of computational cases}

Building density can reflect the vacancy rate within a certain area. It refers to the ratio of the total base area of a building to the total land area within a certain range. In this section, the layout of buildings in a certain district is selected as prototype cases. Five hypothetical building density cases are simulated. The base areas of each residential district in each case are the same, i.e., $160 \mathrm{~m} \times 160 \mathrm{~m}$. The specific layout is shown in Table 1.

Table. 1. Different cases of building density

\begin{tabular}{c|c|c|c|c}
\hline Case & Building volume (m) & Fore-and-aft spacing $(\mathbf{m})$ & Lateral spacing $(\mathbf{m})$ & Building density \\
\hline 1 & $15 \times 25 \times 20$ & 33 & 42.5 & 0.18 \\
2 & $15 \times 30 \times 20$ & 33 & 35 & 0.21 \\
3 & $15 \times 35 \times 20$ & 33 & 27.5 & 0.25 \\
4 & $15 \times 40 \times 20$ & 33 & 20 & 0.28 \\
5 & $15 \times 45 \times 20$ & 33 & 12.5 & 0.32 \\
\hline
\end{tabular}

In the study of the impact of building density on wind environment inside residential buildings, 12 middle-level buildings are set in a residential district. The long edge of a building is changed to change its residential density. The height of a building is set to $20 \mathrm{~m}$. 


\subsection{Domain size and grid generation}

The size of the computational domain should be set to prevent any diverse effect of the surrounding boundaries, such as reverse airflow, which causes the solution to diverge. Several recommendations have been made depending on the case under investigation. Cheng-Hu and Wang [20] used a separation distance between the boundary and building blocks that was thrice the overall depth (or width) of a building and five times its maximum height. However, a trial-and-error process was recommended at the beginning of the study to determine the most appropriate size that would lead to solution conversion using an acceptable mesh size. The domain size $4.8(\mathrm{~W}) \times 3.5(\mathrm{~L}) \times 5(\mathrm{H})$ is determined appropriate for this study, where L denotes the overall length of the building blocks $(160 \mathrm{~m}), \mathrm{W}$ denotes the overall width of the building blocks $(160 \mathrm{~m})$, and $\mathrm{H}$ denotes the uniform height of the buildings $(20 \mathrm{~m})$.

For the implemented mesh size, horizontal and vertical hierarchies are created to ensure reasonable file sizes and computing time. To achieve this objective, a hexagonal nonstructural meshing scheme is adopted. Relatively coarse meshes are commonly used in built environment simulation cases given that a fine mesh is ensured at datum level. However, a high resolution is ensured in the current study because a mesh size limit of $2.2 \times 10^{6}$ cells is maintained.

\subsection{Boundary conditions}

The boundary conditions implemented in this study are the inlet and outflow of the atmospheric boundary layer. To set the velocity in the inlet boundary condition, wind velocity variation along the solution domain height should be considered. This factor is highly dependent on terrain roughness. For example, wind speed is considerably lower in a city terrain than in an open country terrain. The variation of wind velocity with height is given in the following equation:

$$
V_{z}=V_{1.5}\left(\frac{z}{1.5}\right)^{\alpha}
$$

where $V_{z}$ is the mean reference wind speed $(2.8 \mathrm{~m} / \mathrm{s}$ in this study), 1.5 is the datum point height, $V_{1.5}$ is the relative wind speed at pedestrian height, $z$ is the reference height, and $\alpha$ indicates terrain roughness $(0.2$ in the case of an urban terrain). The outlet boundary conditions are set as free-flow boundaries. The top and sides are set as non-sliding wall boundary conditions.

\subsection{Turbulence model and convergence criteria}

In addition to velocity magnitude, the turbulence parameters at the inlet should be defined. These parameters are essential for processing the solution using turbulence models, such as the renormalization group (RNG) k-epsilon model. In this two-equation turbulence model, two separate transport equations are solved to determine the value of the turbulent kinetic energy, $\mathrm{k}$, and its dissipation rate, epsilon. This model is commonly used in simulating buildings where wind environment is highly turbulent. The RNG k-epsilon model is implemented as follows in this study:

$$
\begin{aligned}
& \frac{\partial}{\partial t}(\rho k)+\frac{\partial}{\partial x_{i}}\left(\rho k u_{i}\right)=\frac{\partial}{\partial x j}\left(\alpha_{k} u_{e f f} \frac{\partial k}{\partial x_{i}}\right)+G_{k}+G_{b}-\rho \varepsilon \\
& \frac{\partial}{\partial t}(\rho \varepsilon)+\frac{\partial}{\partial x_{i}}\left(\rho \varepsilon u_{i}\right)=\frac{\partial}{\partial x i}\left(\alpha_{\varepsilon} u_{e f f} \frac{\partial \varepsilon}{\partial x_{i}}\right)+C_{1 \varepsilon} \frac{\varepsilon}{k}\left(G_{k}+C_{3 \varepsilon} G_{b}\right)-C_{2 \varepsilon} \rho G_{k}+\frac{\varepsilon^{2}}{k}-R \varepsilon
\end{aligned}
$$

where $G_{k}$ represents the turbulent kinetic energy $k$ due to the average velocity gradient; $G_{b}$ represents the turbulent kinetic energy $k$ due to the buoyant force; $\alpha_{k}$ and $\alpha_{\varepsilon}$ are the Prandtl numbers that correspond to the turbulent kinetic energy $\mathrm{k}$ and the dissipation rate $\varepsilon$, respectively, where $\alpha_{k}$ and $\alpha_{\varepsilon}$ correspond to $1.39 ; C_{1 \varepsilon}=1.42$; and $C_{2 \varepsilon}=1.68$.

In CFD simulation, a solution is converged when the user-defined convergence criteria are met. The specification of the convergence criteria depends on the problem under investigation and the desired output accuracy. Although a convergence of the scaled residuals to $10^{-5}$ is acceptable in most cases, performing a convergence study to examine the effects of the adopted criteria on the results is highly recommended.

\section{Results analysis and discussion}

\subsection{Case 1: Building density of 0.18}

Fig. 1 presents the wind speed ratio (WSR) and age of air (AA) distribution contour map of Case 1. As shown in the WSR contour map, when the windward area is small and obstructions for back-row buildings are few, the flow of air in a residential district is smooth due to the considerable lateral spacing of left and right buildings. The maximum WSR in the roadway is 1.58 , which decreases gradually along the prevailing wind speed and WSR. Wind speed in the area between two rows of buildings is relatively slow because the front buildings are obstructing the rear buildings. WSR is only approximately 0.5 . A low WSR occurs around the corner of the building, and small wind shadow areas are formed around the building with an MWSR of 0.1 to 0.2 at the corner. A wind shadow zone is present at the leeward side of the last row. The area of the wind shadow zone is acceptable and not excessively large because the building density is moderate.

From the perspective of the AA image, the AA distribution in the entire area is uniform, windward-facing AA is small, and gradually increasing along the prevailing wind direction. The maximum AA appears at the leeward side of the last row of buildings with a maximum value at 260-300 s. The AA values around the building and at the leeward side are high, which is attributed to the existence of a vortex zone, and thus, air stagnant time becomes long. 

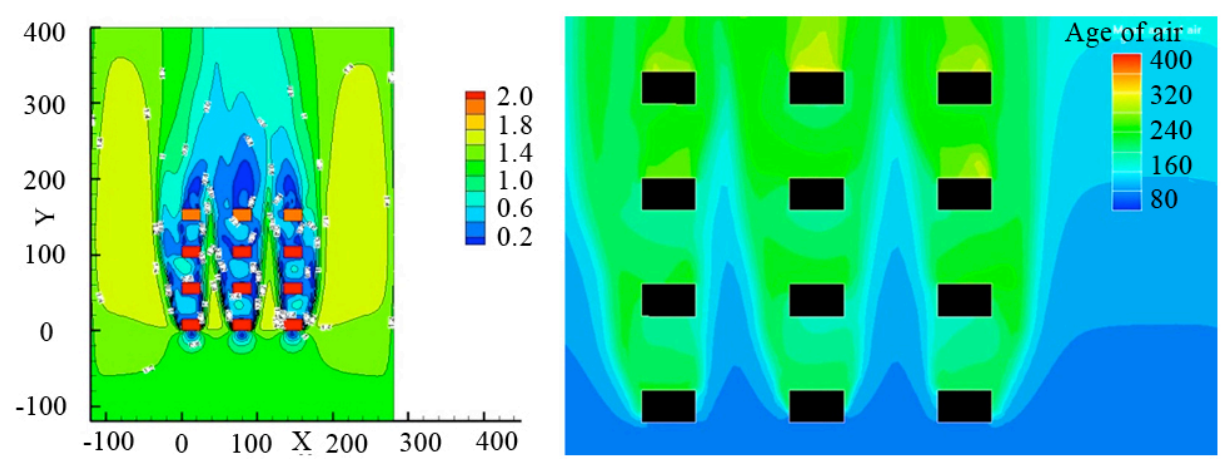

Fig. 1. Wind speed and AA distribution of Case 1

As shown in Fig. 2, WSR distribution in Case 1 is uniform. The area with a WSR higher than 1.0 in the entire building district covers $20 \%$, i.e., $19.03 \%$, thereby showing that the area with high wind speed accounts for a high proportion. The ratio of the minimum WSR $(0 \leqslant$ WSR $\leqslant$ 0.2 ) in the building district is $19.15 \%$, which is mainly distributed around the building. Statistics indicate that the MWSR in the interior of a residential district is 0.56 , which shows that building groups in the residential district have minimal effect on airflow and overall ventilation is relatively smooth.

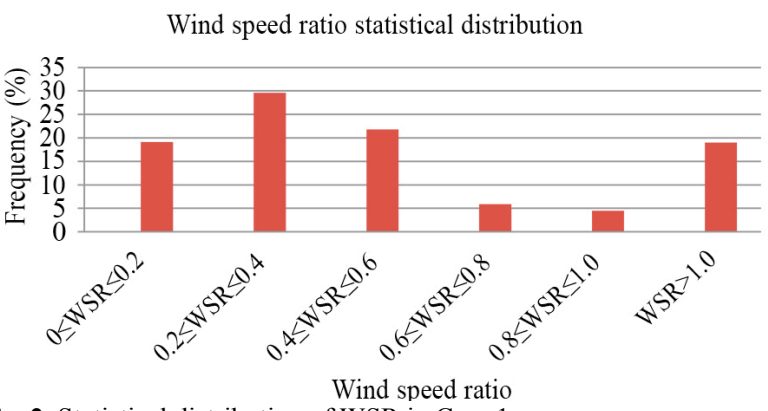

Fig. 2. Statistical distribution of WSR in Case 1

From the AA distribution shown in Fig. 3, the area with the largest AA (AA > $250 \mathrm{~s}$ ) accounts for only $1.4 \%$ of the total region. The air with the largest percentage of AA ranges from $150 \mathrm{~s}$ to $200 \mathrm{~s}$, thereby reaching $45.2 \%$. By contrast, the area with an AA less than 200 s reaches $79.4 \%$, thereby showing that AA within the entire residential district is small and air quality in the district is good. The AA in the building district is $158.48 \mathrm{~s}$, which indicates that air in the district is fresh.

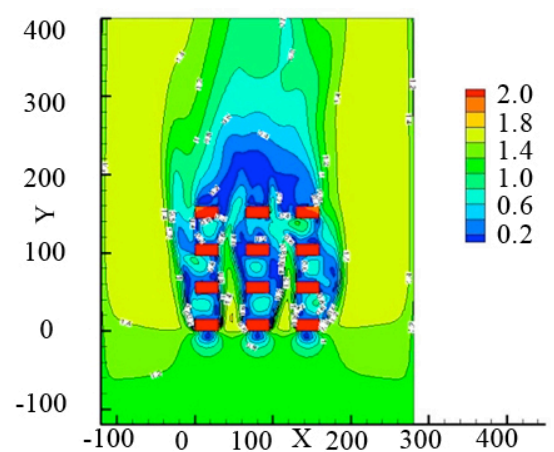

Fig. 4. Wind speed and AA distribution of Case 2

From the WSR statistics provided in Fig. 5, the minimum WSR $(0 \leqslant$ WSR $\leqslant 0.2)$ of building density in Case 2 is $21.46 \%$, which is slightly higher than that in Case $1(19.15 \%)$. The maximum WSR (WSR $>1.0$ ) accounts for $14.23 \%$, which is less than that in Case 1 (19.03\%). The

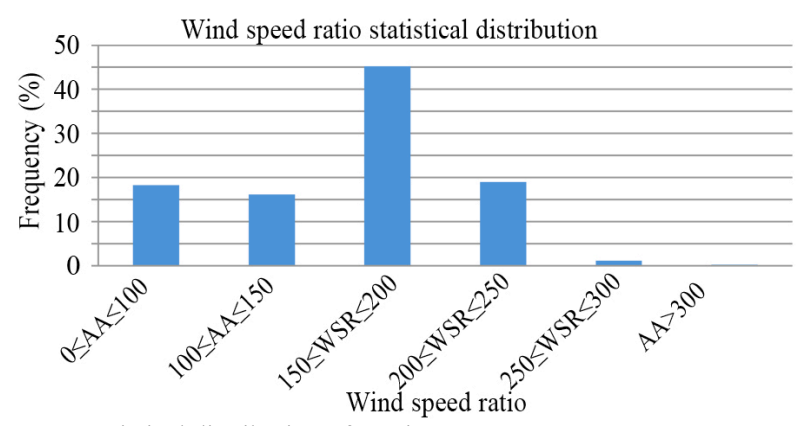

Fig. 3. Statistical distribution of AA in Case 1

\subsection{Case 2: Building density of 0.21}

Fig. 4 presents the WSR contour image and AA distribution of Case 2. As shown in the wind speed contour map, the windward area of a building increases and the spacing between the two sides of the building is reduced with an increase in residential building density, and thus, wind speed is enhanced when an incoming wind stream passes through the roadway. The maximum WSR reaches 1.62. Although wind speed at the entrance of the roadway is increased, wind speed near the last row of buildings is nearly zero due to the increased windward area. A wind shadow area appears around the corner of the building. Simultaneously, a larger wind shadow area is formed at the last row.

From the perspective of the AA image, the AA of the residential district within the region is increased. The maximum AA also appears at the leeward of the last row of buildings. The minimum AA area lies in the passage of two columns of buildings and AA gradually increases along the direction of incoming air flow.

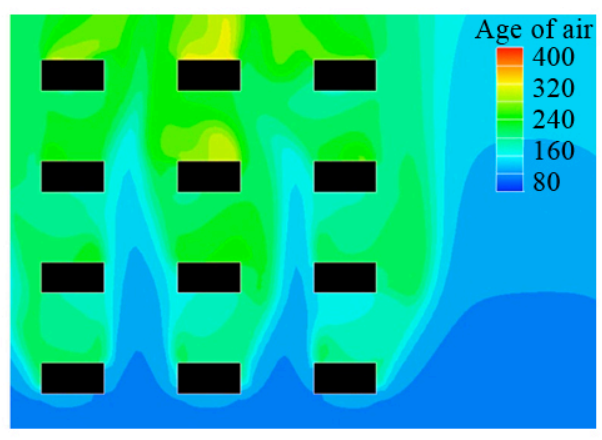

difference between the two values is $4.80 \%$. The mean wind speed of the buildings in the residential district decreases with an MWSR of 0.51 because of increasing building density. 


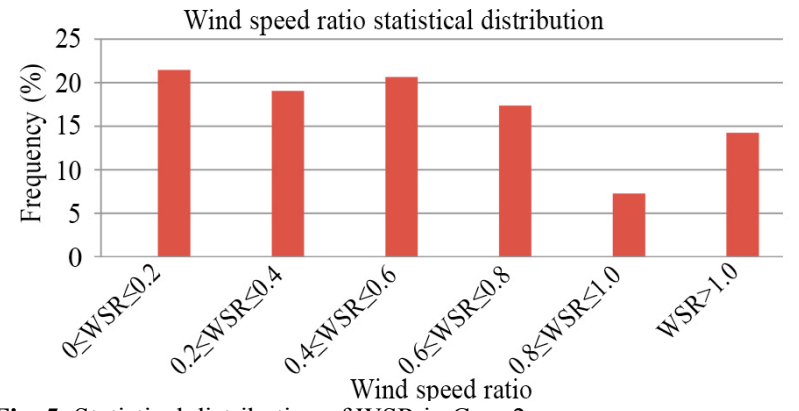

Fig. 5. Statistical distribution of WSR in Case 2

From the AA distribution shown in Fig. 6, the area with the highest AA (AA > $250 \mathrm{~s}$ ), which accounts for $2.14 \%$, is larger than that in Case $1(1.40 \%)$, thereby showing an increase in the area with poor air flow. The area with the smallest $\mathrm{AA}(0 \leqslant \mathrm{AA} \leqslant 100 \mathrm{~s})$ accounts for $16.57 \%$, which is slightly smaller than that in Case $1(18.28 \%)$. This result indicates a decrease in the area with fresh air, with an MAA of $170.12 \mathrm{~s}$.

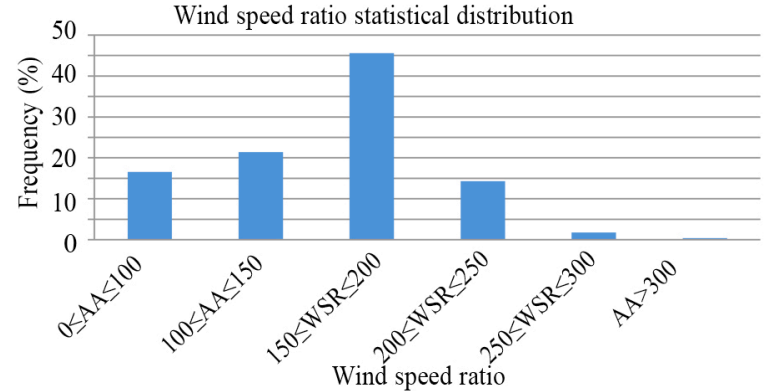

Fig. 6. Statistical distribution of AA in Case 2

\subsection{Case 3: Building density of 0.25}

Fig. 7 presents the WSR contour map and AA distribution of Case 3. As shown in the contour of WSR, the lateral spacing between two buildings is reduced, the MWSR inside the area is decreased, and the area with low wind speed is evidently increased due to the further increase in building density. The buildings have more wind shadow areas at the leeward side and at the corner.

From the AA distribution, the area with the smaller AA appears between the front buildings at the windward side, whereas the area with the largest AA appears around the buildings from the second row to the last row, particularly the corner between the buildings in the last row. AA reaches $350 \mathrm{~s}$.

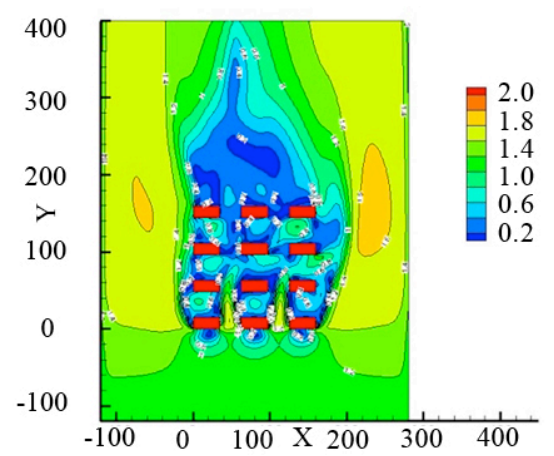

Fig. 7. Wind speed and AA distribution of Case 3

From the WSR shown in Fig. 8, the ratio of the minimum WSR $(0 \leqslant$ WSR $\leqslant 0.2)$ in Case 3 is $25.69 \%$, which is slightly higher than that in Case $2(21.46 \%)$. The maximum WSR (WSR $>1.0$ ), accounts for $6.13 \%$, which is lower than that in Case $2(14.23 \%)$. The difference between the two values is $8.10 \%$. The mean wind speed of buildings in the residential district decreases again with an MWSR of 0.47 due to the further increase in building density.

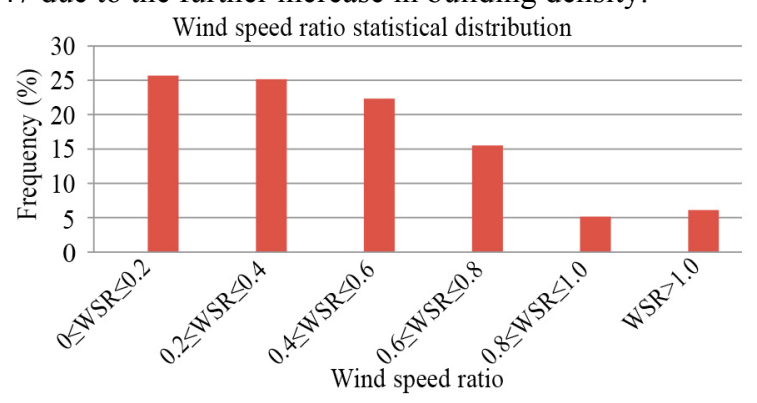

Fig. 8. Statistical distribution of WSR in Case 3

On the basis of the AA distribution shown in Fig. 9, the area with the largest AA (AA > $250 \mathrm{~s}$ ) occupies $8.49 \%$ in Case 3, which is larger than that in Case $2(2.14 \%)$, thereby indicating an increase in the proportion of areas with

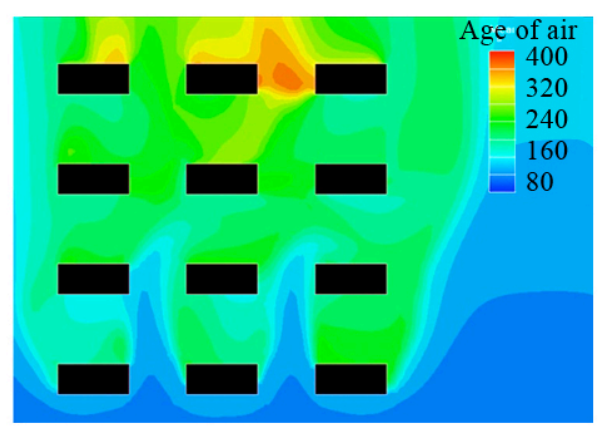

unsmooth air. The area with the smallest AA $(0 \leqslant$ AA $\leqslant$ $100 \mathrm{~s}$ ) accounts for $9.79 \%$, which is smaller than that in Case $2(16.57 \%)$. This result indicates that the proportion of fresh air area is further reduced compared with that in Case 2. AA exhibits an increasing trend, with an MAA of $183.56 \mathrm{~s}$.

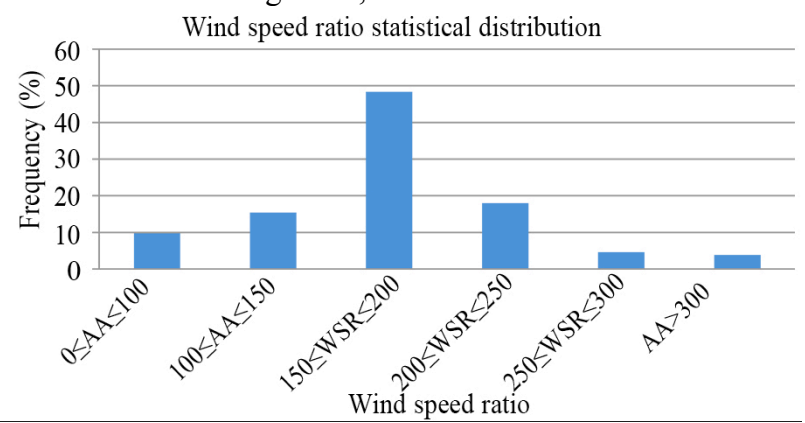

Fig. 9. Statistical distribution of AA in Case 3

\subsection{Case 4: Building density of 0.28}

Fig. 10 presents the contour map of WSR and the distribution of AA of Case 4. An acceleration effect develops with a maximum WSR of 1.73 due to the further narrowing of the roadway between two rows of buildings. However, the lateral spacing between the two buildings is smaller and airflow is further hindered because of the 
increase in building density. Vortex areas are formed between and around buildings, and wind speed in these areas is small. WSR at the leeward side of the last row of buildings is less than 0.1 , which is highly detrimental to the ventilation inside buildings.

From the image of AA distribution, the AA distribution in the residential district in Case 4 changes with the AA of the first row of buildings (approximately $50 \mathrm{~s}$ ) and that of the last row of buildings (approximately $300 \mathrm{~s}$ ). The difference between the two values is $250 \mathrm{~s}$. The leewardfront AA of the last row of buildings reaches the maximum value of approximately $380 \mathrm{~s}$. In such case, providing the fresh air demand of pedestrians is difficult.

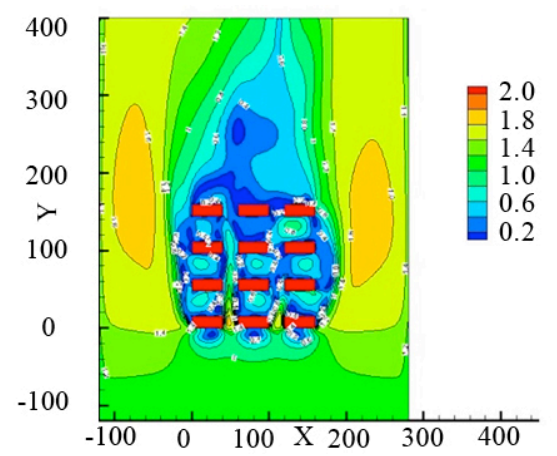

Fig. 10. Wind speed and AA distribution of Case 4

From the WSR distribution shown in Fig. 11, the ratio of the minimum WSR $(0 \leqslant \mathrm{WSR} \leqslant 0.4)$ in Case 4 is $58.82 \%$, which is higher than that in Case $3(51.16 \%)$. The maximum WSR interval (WSR > 1.0) accounts for 3.94\%, which is smaller than that in Case $3(6.13 \%)$. The mean wind speed of the buildings in the residential district decreases further, with an MWSR of 0.43 , due to the increase in building density.

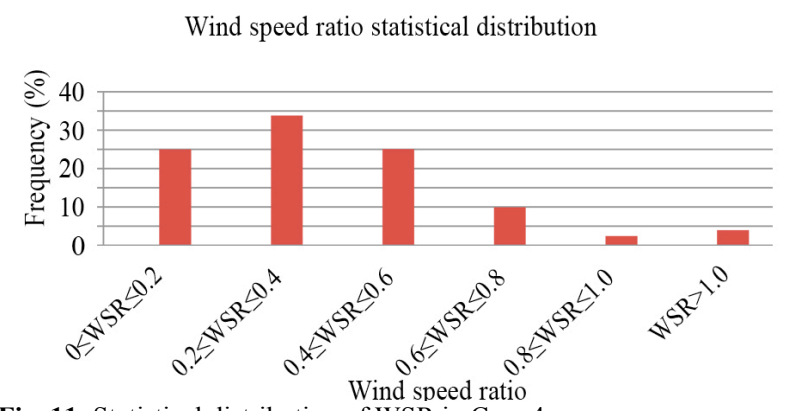

Fig. 11. Statistical distribution of WSR in Case 4

As shown in the AA distribution in Fig. 12, the area with the largest AA (AA $>250 \mathrm{~s})$ occupies $11.93 \%$ of the region in Case 4, which is larger than that in Case $3(8.49 \%)$, thereby increasing the proportion of unsmooth regions by $3.44 \%$. The area with the smallest AA $(0 \leqslant \mathrm{AA} \leqslant 100 \mathrm{~s})$ accounts for $7.16 \%$ of the total region, which is smaller than that in Case $3(9.79 \%)$, thereby indicating that the proportion of fresh air is reduced. The MAA is further increased, i.e., $196.89 \mathrm{~s}$.

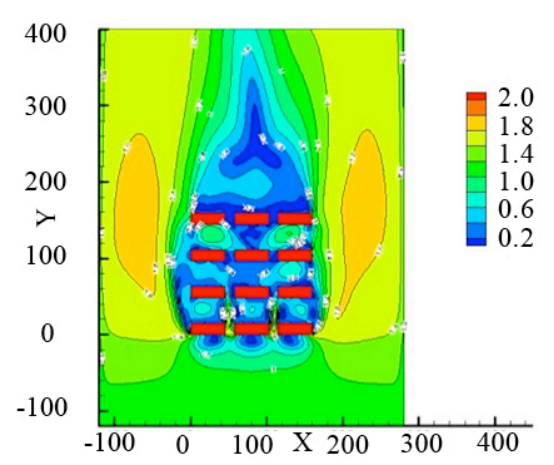

Fig. 13. Wind speed and AA distribution of Case 5.
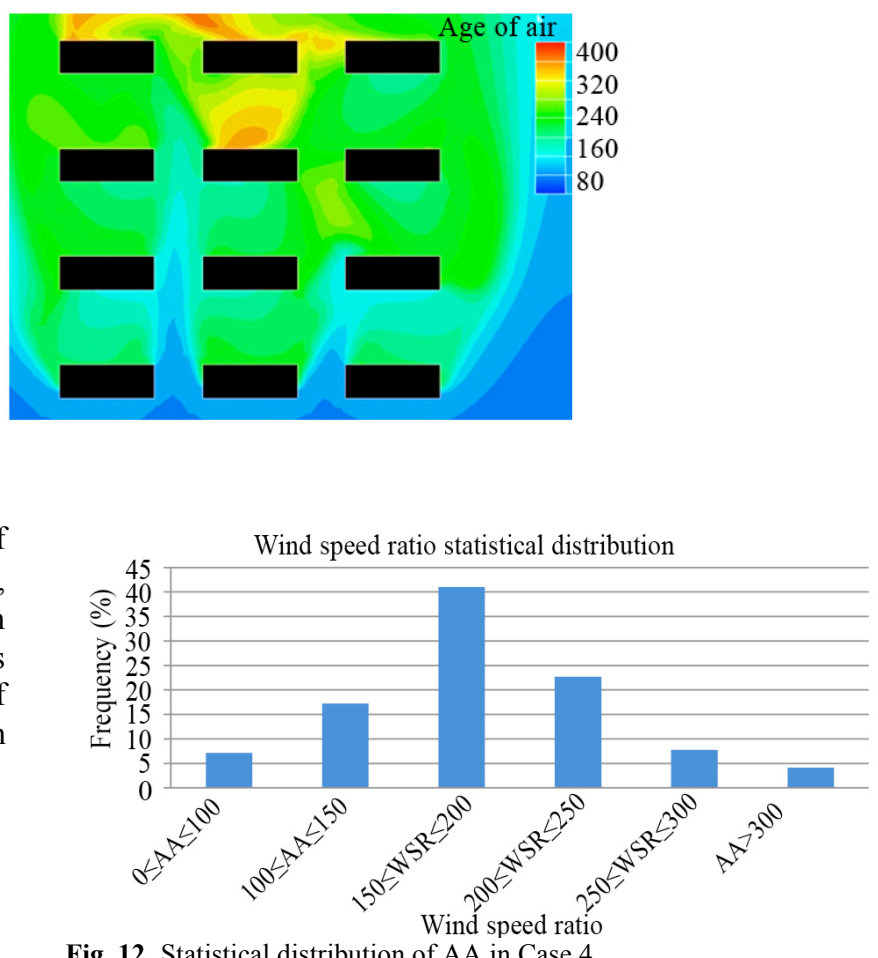

Fig. 12. Statistical distribution of AA in Case 4

\subsection{Case 5: Building density of 0.32}

Fig. 13 presents the WSR contour map and AA distribution of Case 5. As shown in the WSR contour map, the lateral spacing between two buildings is the smallest as building density increases to a maximum of 0.32 . In addition, airflow is hampered the most, thereby forming a scroll area between and around buildings. For example, the leeward side of the last row of buildings is nearly entirely under the shade of the wind, which not only affects outdoor ventilation in the district but is also extremely detrimental to indoor ventilation.

From the distribution of AA, AA around the first two rows of buildings is relatively small, whereas that around the last two rows of buildings is evidently increased. The leeward side and AA at the last row of buildings remain at approximately $440 \mathrm{~s}$ and the area with a large AA increases significantly.

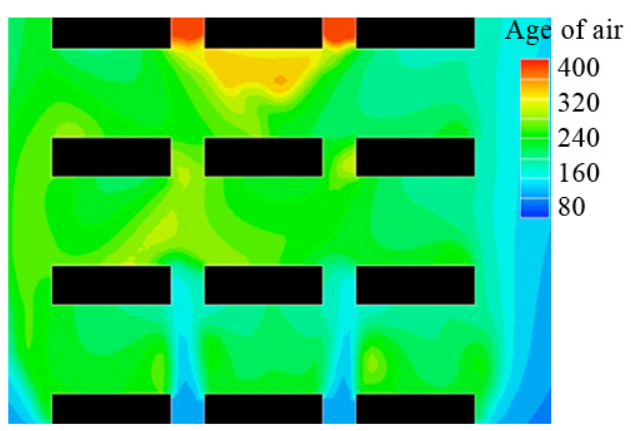


From the WSR distribution in Fig. 14, the ratio of the minimum WSR $(0 \leqslant$ WSR $\leqslant 0.2)$ in Case 5 is $29.84 \%$, which is higher than that in Case $4(24.96 \%)$, thereby indicating that the proportion of the minimum wind speed area increases with an increase in building density. The ratio of the maximum WSR (WSR $>1.0$ ) is $2.87 \%$, which is less than that in Case $4(3.94 \%)$. A narrowing of residential ventilation ducts and a decrease in the proportion of highspeed winds occur due to increasing building density. The MWSR within the district is 0.38 .

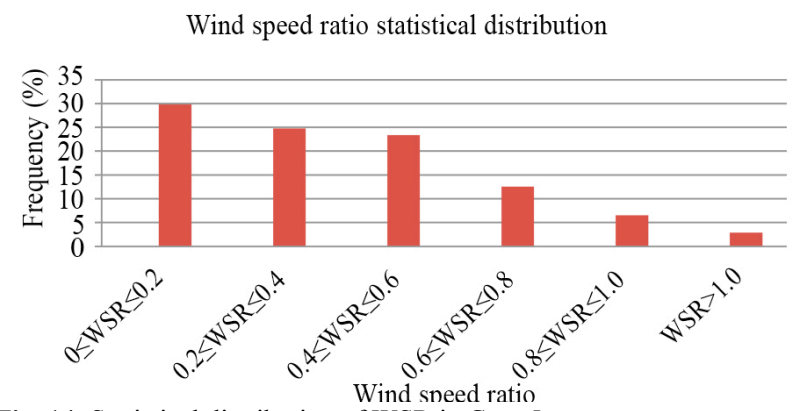

Fig. 14. Statistical distribution of WSR in Case 5

From the AA distribution shown in Fig. 15, the area with the largest AA (MAA > $250 \mathrm{~s}$ ) occupies $23.44 \%$, which is larger than that in Case $4(11.51 \%)$. The area with the smallest AA $(0 \leqslant$ MAA $\leqslant 100 \mathrm{~s})$ accounts for $4.76 \%$, which is smaller than that in Case $4(7.16 \%)$, thereby indicating that the proportion of fresh air area is further reduced. The MAA is further increased, i.e., $217.11 \mathrm{~s}$.

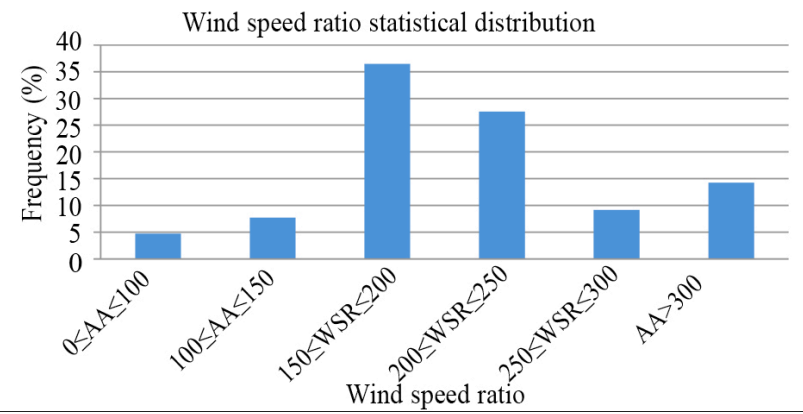

Fig. 15. Statistical distribution of AA in Case

After simulating and analyzing the five cases of building density, the WSR and AA data simulated in each wind environment case are processed averagely. The MWSR and MAA results for the five cases of residential districts $(160 \mathrm{~m}$ $\times 160 \mathrm{~m}$ ) are presented in Table 2 .

Table 2. Mean wind speed ratio (MWSR) and mean age of air (MAA) in the five building density cases

\begin{tabular}{c|c|c|c}
\hline Case & Building density & MWSR & MAA \\
\hline Case 1 & 0.18 & 0.56 & 158.48 \\
Case 2 & 0.21 & 0.51 & 170.12 \\
Case 3 & 0.25 & 0.47 & 183.56 \\
Case 4 & 0.28 & 0.43 & 196.89 \\
Case 5 & 0.32 & 0.38 & 217.11 \\
\hline
\end{tabular}

The data in Table 2 are inputted into the statistical software SPSS for unary linear analysis. After statistical processing, the relationship between the different building densities of the residential districts and the overall MWSR and MAA is shown in Figs. 16 and 17, respectively.

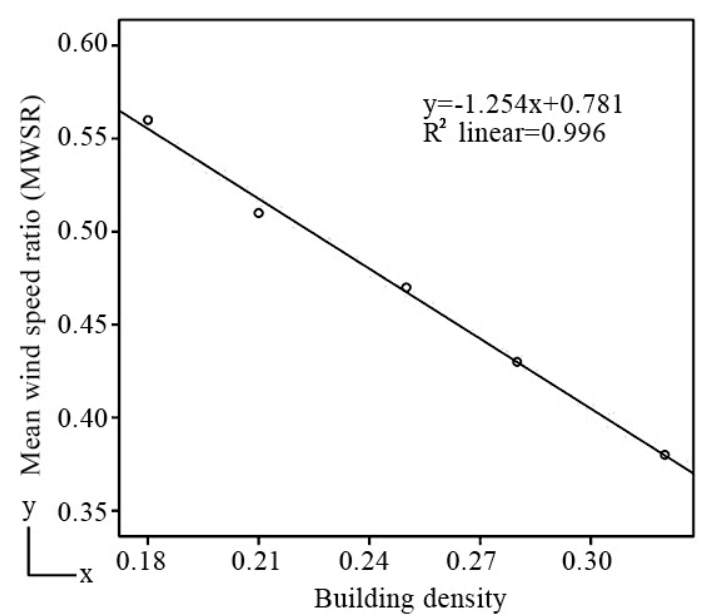

Fig. 16. Relationship between building density and MWSR

The relationship between building density and MWSR is:

$y=-1.254 x+0.781$

Formula: y-MWSR

$\mathrm{X}$-building density

From the relationship between building density and MWSR, a significant negative correlation is observed between residential building density and outdoor MWSR. That is, when building density is high, MWSR is small; , when building density is low, MWSR is large.

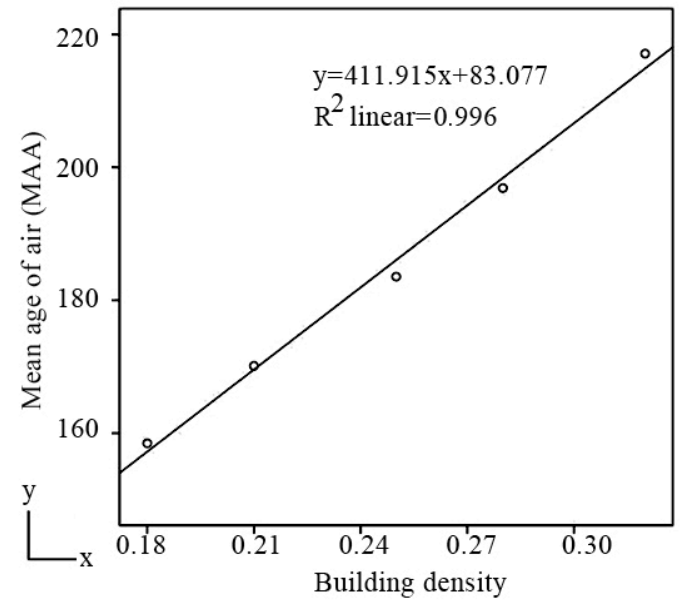

Fig. 17. Relationship between building density and MAA

The relationship between building density and MAA is

$y=411.915 x+83.077$

Formula: y-MAA x-building density

As indicated in the relationship between building density and MAA, residential building density is significantly and positively correlated with outdoor MAA. That is, when building density is high, MAA is large; when building density is low, MAA is small. 


\subsection{Tactical research for improving wind environment in residential buildings}

The influence of building density on wind environment in buildings are simulated and analyzed. Building density for the planning and design of residential districts may have an impact on pedestrian comfort mainly in the following points:

(1) Pedestrians may feel uncomfortable in both residential building access points because the pipe-tube effect increases with wind speed.

(2) Wind speed at the leeward side and around the corners of buildings is low, and thus, AA values in these areas tend to be high. Therefore, the activities of people in these areas may be less comfortable.

(3) In the process of residential planning and design, excessive building density will adversely affect the overall outdoor ventilation of residential districts and reduce air quality and the outdoor comfort of pedestrians.

This study proposes the following tactics based on the information that the building density of residential districts affect wind environment.

(1) To avoid safety problems caused by the canyon effect between the entrance channels of residential districts, the lateral windward distance of building windward surface may be appropriately increased to reduce the wind effect of the roadway. For a roadway with a high density of residential buildings on both sides, a large area of green or windproof wall can be built to block high-speed airflow, and thus, reduce the initial wind speed at the entrance of buildings and alleviate the wind effect of roadways.

(2) In the main air duct overhead part of buildings, airflow can be guided into the leeward side of buildings.

(3) Residential districts should not set an excessively high building density during the planning and design stages. In certain building density values, the reasonable layout of building groups should be adopted according to the prevailing local wind direction.

\section{Conclusions}

To quantitatively investigate the influence of residential building density on residential wind environment, this study established five residential models under different residential building density conditions using computer numerical simulation and then analyzed the effect of different residential building densities on outdoor wind environment. WSR and AA changes were recorded and analyzed. The following conclusions can be drawn.
(1) With the continuous increase in residential building density, the proportion of low WSR gradually becomes smaller and that of high WSR gradually decreases because of the gas pipe effect and the maximum WSR becomes higher.

(2) The size of the windward opening area of a residential district significantly impacts the distribution of outdoor wind environment within the district. When the opening area is large, the ventilation is good. Roadways between buildings that are too narrow will result in local high winds and negatively impact pedestrians.

(3) With an increase in residential building density, the MAA value of a residential area and proportion of the area occupied by the large AA area increase.

(4) The distribution of AA is closely related to the distribution of WSR, and the two factors exhibit a negative correlation. When WSR is high, AA is small. By contrast, when WSR is low, AA is large.

(5) From the MWSR and MAA of the entire district, the building density of residential areas is modified by changing the opening size of the windward area. Outdoor MWSR is negatively correlated with building density. MAA is positively correlated with building density. As building density increases, the MWSR within the area is reduced and MAA is increased, which affects the comfort of people when performing outdoor activities.

In this study, WSR and AA were considered to evaluate the variation in outdoor wind environment in several residential districts with different building densities and to directly reflect the impact characteristics of residential areas with different building densities on outdoor wind environment in the form of data statistics. This study provides theoretical guidance for the planning, design, and construction of settlements.

However, due to the lack of wind environment measurement or wind tunnel experiment data of residential districts with different building densities, computer numerical simulations may lead to deviations in the simulated parameter settings from the actual values. Therefore, future research can focus on determining the influence of building density in different settlements on wind environment by using actual measurements. An accurate response to the influence of building density on outdoor wind environment requires further research.

This is an Open Access article distributed under the terms of the Creative Commons Attribution License

\section{References}

1. Huang L., Miao J., Liu Y., "Temporal and Spatial Variations of Tianjin Urban Heat Island Effect". Journal of Nanjing Institute of Meteorology, 35( 5), 2012, pp.620-632.

2. Kang H., Zhu B., Zhu T., "A Case Study of the Urban Heat Island Effect in Suzhou-Wuxi Changzhou". Journal of Nanjing Institute of Meteorology, 37( 4), 2014, pp.432-440.

3. Zheng S., Quan Y., Dong Z., "Study on residential wind environment of strong wind area". Environmental Protection Science, 39(3), 2013, pp.64-68.

4. Yi Q., Tao S., Zhan Q., "Research on outdoor wind environment of building groups based on computer simulation". Computer Modelling and New Technologies, 18 (10), 2014, pp.362-367.
5. Huang Z., Li L., Zhao L., "Quantitative analysis for impact of first floor overhead on natural ventilation under different building densities and plot ratios". $H V \& A C, 46(9), 2016$, pp.124-127.

6. Guo F., Zhu P., Wang S., "Quantitative study of correlation of urban morphology and wind environment in a high density area: A case of Dalian”. Academic Journal, 11(16), 2017 Supplement 1, pp.14-17.

7. T. K., M. Miura, Y. Tominaga, A. Mochida, "Wind tunnel tests on the relationship between building density and pedestrian-level wind velocity: development of guidelines for realizing acceptable wind environment in residential neighbourhoods". Building and Environment, 43 (10), 2008 , pp.1699-1708. 
8. A. Zhang, G. Cuilan, L. Zhang, "Numerical simulation of the wind field around different building arrangements". Journal of wind engineering and industrial aerodynamics, 93(12), 2005, pp. 891904.

9. Xiaoyu Y., Wei Z., Kanozuri Hokao, "Numerical research of layout effect on wind environment around high-rise buildings". Architectural Science Review, 56(4), 2013, pp.272-278.

10. Yafeng Gao, Runming Yao, Baizhan Li, "Field studies on the effect of built forms on urban wind environments". Renewable Energy, 46(5), 2012, pp.148-154

11. Gong chen, Wang xin, "Research on wind environment for urban residential district in different building layouts". Building Science, 30(7), 2014, pp.6-12.

12. Fang P., Shi J., Wang Q., "Numerical study on wind environment among tall buildings in Shanghai Lujiazui Zone". Journal of Building Structures, 34(9), 2013, pp.104-111.

13. Feng Y., "Investigating wintertime pedestrian wind environment and user perception in dense residential neighbourhood in a city of hot-summer and cold-winter climate zone, China". Indoor and Build Environment, 26(3), 2017, pp.392-408.
14. Ghada Elshafei, Abdelazim Negm, Mahmoud Bady, "Numerical and experimental investigations of the impacts of windowparameters on indoor natural ventilation in a residential building". Energy and Buildings, 141, 2017, pp.321-332.

15. Xing Shi, Yiyu Zhud, Jin Duanc, "Assessment of pedestrian wind environment in urban planning design". Landscape and Urban Planning, 140, 2015, pp.17-28.

16. Li Tanga, Marialena Nikolopoulou, Fu-yun Zhao, Nan Zhang, "CFD modeling of the built environment in Chinese historic settlements". Energy and Buildings, 55(12), 2012, pp.601-606.

17. C.W. Tsang, K.C.S. Kwok, P.A. Hitchcock, "Wind tunnel study of pedestrian level wind environment around tall buildings: Effects of building dimensions, separation and podium". Building and Environment, 49(3), 2012, pp.167-181.

18. Akashi Mochida, Isaac Y.F. Lun, "Prediction of wind environment and thermal comfort at pedestrian level in urban area". Journal of Wind Engineering and Industrial Aerodynamics, 96(10), 2006, pp.1498-1527.

19. P. J. Jones, D. Alexander, J. Burnett, "Pedestrian Wind Environment Around High-Rise Residential Buildings in Hong Kong". Indoor and Build Environment, 13(4), 2004, pp.259-269.

20. H. Cheng-Hu, F. Wang, "Using a CFD approach for the study of street-level winds". Build. Environ. 40(5), 2005, pp.617-631. 